\title{
Quality Assurance for Interventional Pain Management Procedures
}

YiLi Zhou, MD, PhD, Fred A. Furgang, MD, and YanPing Zhang, PhD

Background: Over the last decade various guidelines for quality assurance in pain medicine have been published for cancer pain, acute postoperative pain and other pain conditions. However, quality assurance for interventional pain management procedures has yet to be addressed. An interventional pain program should at least evaluate 1) efficacy of pain relief; 2) complication rate; and 3) patient satisfaction.

Objective: This study was designed to monitor the quality of interventional pain management procedures in a university teaching hospital.

Study Design: A prospective survey.

Methods: From January 1, 2004, to June 30,2004 , the quality of interventional pain management procedures in a university teaching hospital in Miami, Florida was monitored. Questionnaires assess-

The purpose of a Quality Assurance program in pain medicine is to provide a mechanism for reviewing patient-related activity on a continuous basis in order to monitor, analyze, and improve patient outcomes. Over the last two decades quality assurance in pain management has gained increasing attention from the medical community, as well as from governmental agen-

From: Comprehensive Pain Management of North Florida, Gainesville, Florida; Jackson Memorial Hospital Pain Management Center, University Of Miami Miller School Of Medicine; and Department Of Anesthesiology, University of Miami Miller School Of Medicine, Miami, Florida Address Correspondence: YiLi Zhou, MD, PhD, 10303 SW $48^{\text {th }} \mathrm{Pl}$, Gainesville, FL 32608 Email: Yilizhoumd@yahoo.com

Conflict of Interest: None

Disclaimer: There was no external funding in preparation of this manuscript. Manuscript received on: 02/03/2006 Revison submitted on: 03/08/2006 Accepted for publication on 03/09/2006 ing immediate pain relief, patient satisfaction, and complications were provided to each patient and physician immediately after completion of each procedure. Data was collected before patients were discharged.

Results: A total of 566 patients with a mean age of 52.9 years participated in the survey. Interventional pain management procedures included epidural steroid injections, facet joint blocks, transforaminal epidural injections, sympathetic nerve blocks, lumbar discography, nucleoplasty, percutaneous disc decompression, spinal cord stimulator trial, and intravenous regional blocks, etc. Among 528 patients who reported their pain scores before and after procedures, 487 (92\%) patients reported various degrees of pain relief immediately following their procedures. The

cies, which oversee pain management in hospitals and other health care facilities. The Joint Commission on Accreditation of Healthcare Organizations (JCA$\mathrm{HO}$ ) published an official statement for the purpose of improving the quality of pain management in 2003. U.S. hospitals(1) and other patient care facilities, such as nursing homes $(2,3)$, have subsequently utilized more resources and efforts to improve the quality of pain management. Various guidelines, consensus statements and suggestions for quality assurance have been published for pain management in patients with cancer (4), acute postoperative pain (5), obstetrical pain (6), palliative care $(7,8)$, intensive care units (9), rheumatologic pain $(10,11)$ and in managed care settings (12). Quality assurance projects have been applied in multiple health care facilities $(2,13,14)$. As a result, a better quality of care has been re- average pain score decreased 4.7 on a 0 to 10 scale after treatment $(p<0.001)$. No major complications were reported for this group of patients. Among 442 patients who answered the question regarding satisfaction, 406 (91.8\%) were satisfied, or highly satisfied, with the immediate outcome of their procedures.

Conclusion: The results of the current study indicate that quality assurance of interventional pain management procedures in terms of immediate pain relief following the procedure, low complication rate, and high patient satisfaction can be achieved through application of a quality assurance program.

Key Words: Interventional pain management procedures, quality assurance, efficacy, patient safety, patient satisfaction

ported as indicated by a significantly reduced prevalence of pain in institutionalized patients, and higher levels of patient satisfaction(2) .

Interventional pain management procedures have been growing rapidly since 1990 and have become one of the major tools in treating chronic pain (15). The National Uniform Claims Committee (NUCC) defined interventional pain management as the discipline of medicine devoted to the diagnosis and treatment of pain and related disorders with the application of interventional techniques in subacute, chronic, or intractable pain, independently or in conjunction with other modalities of treatment. In 2003, CMS designated specialty code "-09" for interventional pain management. It has been estimated that among Medicare recipients, the frequency of interventional pain management procedures in- 
creased by 95\% from 1998 to 2003 (15). The American Society of Interventional Pain Physicians (ASIPP) has developed evidence-based guidelines for interventional pain management procedures for the purpose of improving compliance and quality of care (16). Numerous reports have been published to investigate the long-term efficacy of interventional pain management procedures; these studies have provided critical evidence of their usefulness. However, literature regarding the application of quality assurance techniques to interventional pain management procedures is scant. As a part of the quality assurance program at Jackson Memorial Hospital's Pain Management Center (PMC) we evaluated the results of our interventional pain management procedures program from January 1, 2004, to June 30, 2004. We evaluated 1) efficacy of pain relief; 2) patient safety or complication rates; and 3) patient satisfaction.

\section{Methods}

From January 1, 2004, to June 30, 2004 , we monitored the quality of interventional pain management procedures in the pain management center (PMC) of Jackson Memorial Hospital, the primary teaching hospital of the University of Miami Miller School of Medicine. Exclusion criteria are illustrated in Table 1.

To ensure the quality of care, the following steps were taken before, during and after interventional pain management procedures: 1) each patient was seen by a resident or fellow under the direct supervision of an attending physician; 2) a diagnosis was formulated; 3) an interventional pain man-

Table 1. Conditions unfavorable for interventional pain procedures

Blood pressure $>160 / 95$

Any sign or symptom of infection

Coumadin within 5 days or clopidogrel (Plavix) within 7 days

Solid food consumption within 6 hours or liquids within 2 hours

Failure to bring along an accompanying adult agement procedure was prescribed as indicted by the patient's diagnosis; 4) each procedure was explained to the satisfaction of the patient; 5) a pre-procedure protocol was handed out to each patient; 6 ) on the patient's return to the PMC for their procedure, vital signs, pain severity, medications, possible

contraindications, diagnosis, name and site of the procedure were re-checked prior to taking patients into the procedure room; 7) procedures were rescheduled if any of the exclusion criteria were present; 8) intravenous access was obtained for all cervical procedures, sympathetic blocks, discography,

\section{POST-PROCEDURE SURVEY}

(Please circle your answers)

1. Were you treated courteously by our staff before, during and after the procedure?
(a) Yes
(b) No

2. Select the phrase that indicates how satisfied or dissatisfied you are with the results of your pain overall.
(a) Very satisfied
(b) Satisfied
(c) Slightly satisfied
(d) Slightly dissatisfied
(e) Dissatisfied
(f) Very dissatisfied

3. Do you have any undesirable reaction resulting from the procedure?
(a) Yes
(b) No If yes, please explain...

Were the procedure and the consent adequately explained to you?
(a) Yes
(b) No If not, please explain...

5. Do you understand what to expect after the procedure and how to contact the clinic physician if you have a problem?

(a) No instructions were given

(b) Instructions are unclear to me or I forget

(c) Instructions are somewhat clear

(d) Instructions are absolutely clear

6. On this scale, how much pain did you have during the procedure?

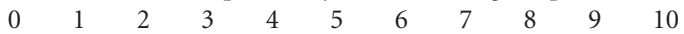

7. On this scale, how uncomfortable (other than pain) were you during the procedure?

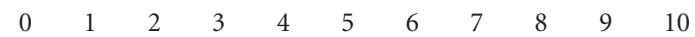

On this scale, how much anxiety did you have during the procedure?

$$
\begin{array}{lllllllllll}
0 & 1 & 2 & 3 & 4 & 5 & 6 & 7 & 8 & 9 & 10
\end{array}
$$

9. On this scale, how much pain were you having just before the procedure?

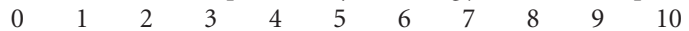

10. On this scale, how much pain are you having now, following the procedure?

$$
\begin{array}{lllllllllll}
0 & 1 & 2 & 3 & 4 & 5 & 6 & 7 & 8 & 9 & 10
\end{array}
$$

List all procedures that you had done today

(a)

(b)

12. Assuming that these procedures helped your pain, would you be willing to undergo them again, sometime in the future?
(a) Yes
(b) No, if not, explain:

Fig. 1. Post-procedure survey of interventional pain management procedures for patients 
disc decompression, spinal cord stimulator trial and vertebroplasty procedures; light intravenous sedation with 1-2 mg of midazolam was only administered to those patients undergoing disc decompression, discography, spinal cord stimulator trial and vertebroplasty; 9) a "time out" was called immediately prior to the interventional pain management procedures to confirm the patient's name, diagnosis, type and site of the procedure; 10) during the procedure, blood pressure, heart rate, pulse oximetry, and EKG were continuously monitored; fluoroscopy was used to guide the needle placement for all spine procedures; 11) each procedure was performed by a resident or fellow under the direct supervision of an attending physician; 12) and upon completion of interventional pain management procedure(s), patients were sent to a recovery area and observed for at least 30 minutes prior to discharge.

Immediately following interventional pain management procedure(s), patients were asked to complete a list of quality assurance questionnaires (Fig. $1)$. There were a total of 12 questions grouped into three categories: 1) efficacy of each procedure. 2) adverse effects experienced during and immediately after the procedure(s), and 3) patient satisfaction. Data was collected before patients were discharged home. Physicians completed a separate quali-

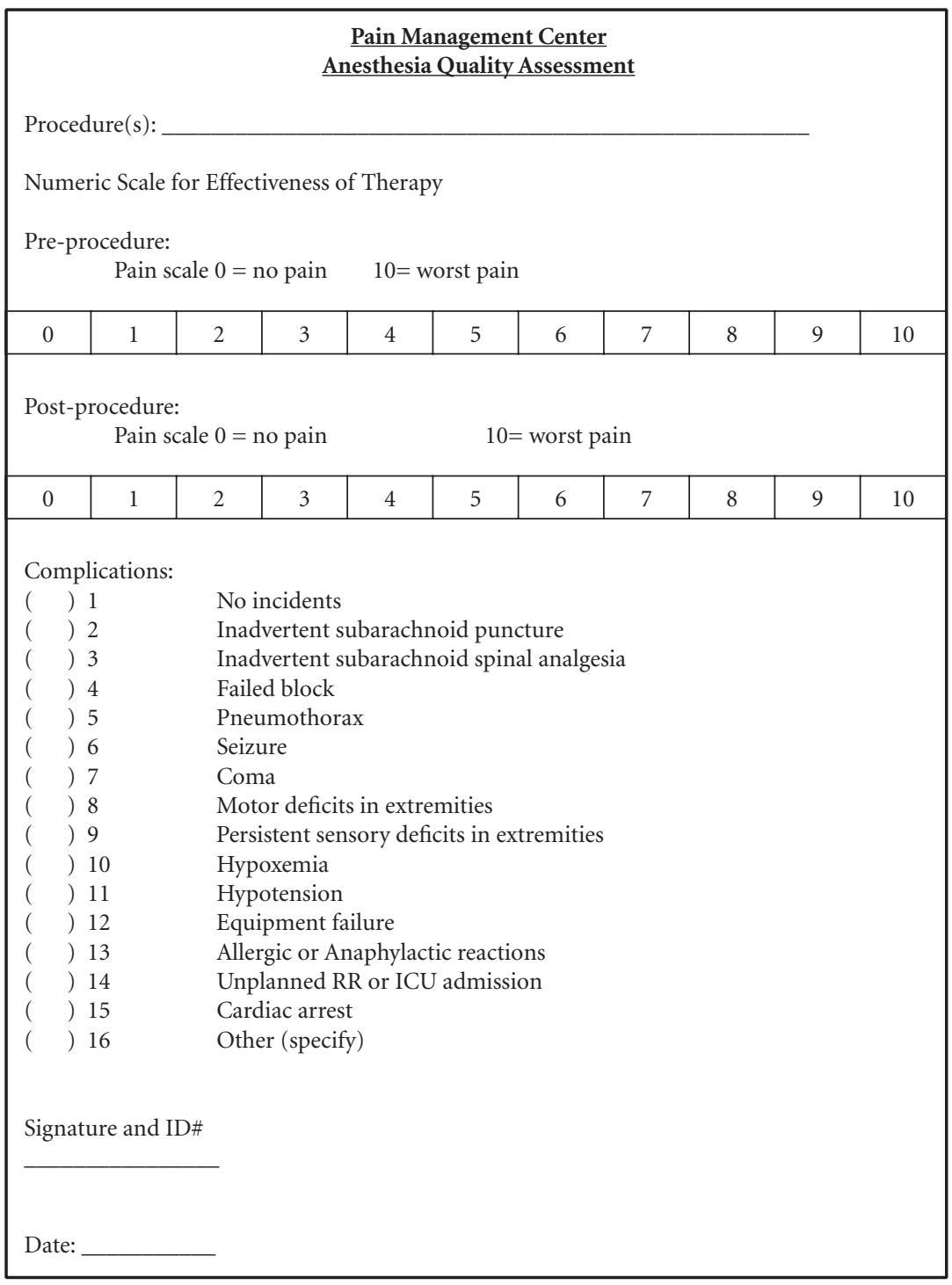

Fig. 2. Post-procedure survey for physicians

ty assurance worksheet regarding efficacy of the procedure and adverse events (Fig. 2).

Patient's pain score immediately prior to, and following the interventional pain management procedures was determined to evaluate the short-term efficacy of treatment. Pain was assessed by an 11-point Numeric Pain Scale $(0=$ no pain and $10=$ worst pain).

To evaluate patient satisfaction, they were asked how they were treated by the staff (Q1); how satisfied they were with the results of the procedure (Q2); whether the procedure and the consent were adequately explained to them (Q4); and did they understand what to expect after the procedure and how to contact the physician (Q5).

For discomfort associated with each procedure, patients were asked to rate the level of overall discomfort, as well as pain and anxiety during the procedure(s) on a 0 to 10 scale.

\section{Statistical Analysis}

Unpaired $t$ test was used to measure significance of pain scores before and after procedures. Correlation between pain during the procedure and degree of pain relief after the procedures was also tested with Wilcoxon matched-pairs test (by using the Prism 3 statistic software).

\section{RESULTS}

A total of 566 patients (male: 262; female: 304; mean age: 52.9 years old) had the interventional pain management procedures during the period of survey. All of these patients participated in the survey. Patients were involved in a wide variety of pain relieving procedures as listed in Table 2.

\section{Effectiveness}

Eight patients had lumbar discography; since lumbar discography is a provocative diagnostic procedure, these patients were excluded from statistical tests for pain relief. Of the remaining 528 patients who reported their pain scores both before and after their interventional pain management procedures, 487 (92\%) had various degrees of pain relief immediately following their interventions. Mean pain score 
Table 2. Procedural characteristics

\begin{tabular}{|c|c|}
\hline Name of Procedure & Frequency \\
\hline Bier block & 4 \\
\hline Celiac plexus block & 4 \\
\hline Lumbar disc decompression & 5 \\
\hline Discography & 8 \\
\hline Cervical epidural injection & 37 \\
\hline Caudal epidural injection & 24 \\
\hline Lumbar epidural injection & 191 \\
\hline Thoracic epidural injection & 5 \\
\hline Cervical facet joint block & 5 \\
\hline Cervical facet radiofrequency & 5 \\
\hline Lumbar facet block & 39 \\
\hline Lumbar facet radiofrequency & 6 \\
\hline Gasserian Ganglion block & 5 \\
\hline Hip joint injection & 2 \\
\hline L2 rami communicans block & 5 \\
\hline L2 rami communicans radiofrequency & 2 \\
\hline Lumbar sympathetic block & 22 \\
\hline Nucleoplasty & 3 \\
\hline Occipital nerve block & 8 \\
\hline Orbital nerve block & 2 \\
\hline Pyriformis muscle injection & 10 \\
\hline Percutaneous adhesiolysis & 13 \\
\hline Suprascapular nerve block & 6 \\
\hline Saphenous nerve block & 2 \\
\hline Spinal cord stimulator trial & 8 \\
\hline Stellate ganglion block & 8 \\
\hline Sacroiliac joint injection & 36 \\
\hline Sphenopalatine ganglion block & 2 \\
\hline Splanchnic nerve block & 2 \\
\hline Cervical transforaminal epidural injection(s) & 3 \\
\hline Lumbar transforaminal epidural injection(s) & 78 \\
\hline Trigger point injection(s) & 11 \\
\hline Vertebroplasty & 5 \\
\hline Total & 566 \\
\hline
\end{tabular}

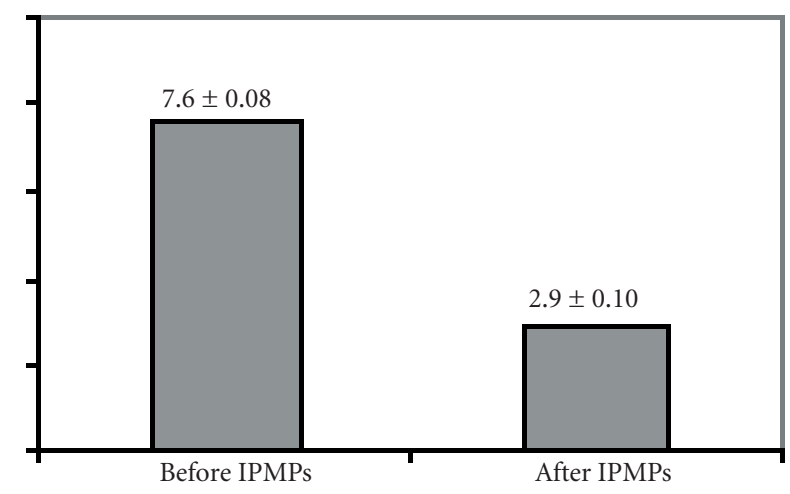

Fig. 3. Immediate pain score (mean $\pm S E$ ) change before and after interventional pain management procedures $(\mathrm{p}<0.001)$ was $7.58 \pm 0.08$ before, and $2.87 \pm 0.10$ after interventional pain management procedures ( $\mathrm{t}=35.75, \mathrm{p}=<0.0001$ ) (Fig. 3). Average pain score decreased 4.7 after treatments. Forty-one (8\%) patients reported no change or increased pain after interventional pain management procedures.

There was a negative relationship between pain during interventional pain management procedures and the degree of pain relief after interventional pain management procedures $\left(\mathrm{R}^{2}=0.01431, \mathrm{p}=0.013\right)$. Patients who experienced more pain during the interventional pain management procedures were more likely to have less pain relief following treatment.

\section{Adverse Effects and Patient Safety}

Eight patients (1.4\%) had fluctuations of blood pressure and heart rate during procedures, which required immediate action. Four patients $(0.7 \%)$ had blood pressure elevation to over 200/100 mmHg during interventional pain management procedures; these procedures were canceled. The other four patients developed bradycardia and/or hypotension during the procedures. All patients were treated immediately and stabilized without long-term consequences (Table 3 ). A 37-year-old female developed chest pain immediately after a lumbar epidural steroid injection; her vital signs remained normal during the procedure. She was sent to the emergency room where tests ruled out myocardial infarction. A 48-year-old male had excessive cutaneous bleeding during a thoracic epidural steroid injection. Two patients had acute migraine attacks prior to lumbar epidural steroid injection. Procedures were canceled for all these three patients. A Gasserian ganglion block, a cervical epidural steroid injection, and a lumbar facet joint block had to be canceled due to patient discomfort.

No major adverse events defined as wrong patient, wrong needle insertion site, inadvertent intrathecal injection, intravascular injection, seizure, infection, paralysis, or death were reported in any patient.

Patients were NPO for six hours 
Table 3. Cardiovascular Instability during interventional pain management procedure(s)

\begin{tabular}{|l|l|l|l|l|}
\hline \multicolumn{1}{|c|}{ Age } & Gender & \multicolumn{1}{|c|}{ Procedure } & \multicolumn{1}{|c|}{ Event } & \multicolumn{1}{c|}{ Action } \\
\hline 55 & F & Lumbar epidural steroid injection & BP 218/122 during procedure & Procedure canceled \\
\hline 58 & M & Lumbar sympathetic block & BP 218/122 during procedure & Procedure canceled \\
\hline 55 & M & Lumbar epidural steroid injection & BP 218/122 during procedure & Procedure canceled \\
\hline 49 & M & Lumbar epidural steroid injection & BP 218/122 during procedure & Procedure canceled \\
\hline 30 & M & $\begin{array}{l}\text { Cervical transforaminal epidural } \\
\text { injection }\end{array}$ & $\begin{array}{l}\text { HR below } 50 / \mathrm{m} \text { and BP below } \\
90 / 40 \mathrm{mmHg}\end{array}$ & $\begin{array}{l}\text { Procedure canceled. BP and HR return to } \\
\text { normal within 2 min. }\end{array}$ \\
\hline 47 & M & Cervical epidural steroid injection & BP 80/50 mmHg, HR 50/m & $\begin{array}{l}\text { One litter of normal saline and } 5 \mathrm{mg} \text { of } \\
\text { ephedrine were given. procedure canceled }\end{array}$ \\
\hline 55 & F & Lumbar epidural steroid injection & HR below 50/m & Procedure canceled \\
\hline 28 & M & Lumbar epidural steroid injection & Mild hypotension & Procedure completed \\
\hline
\end{tabular}

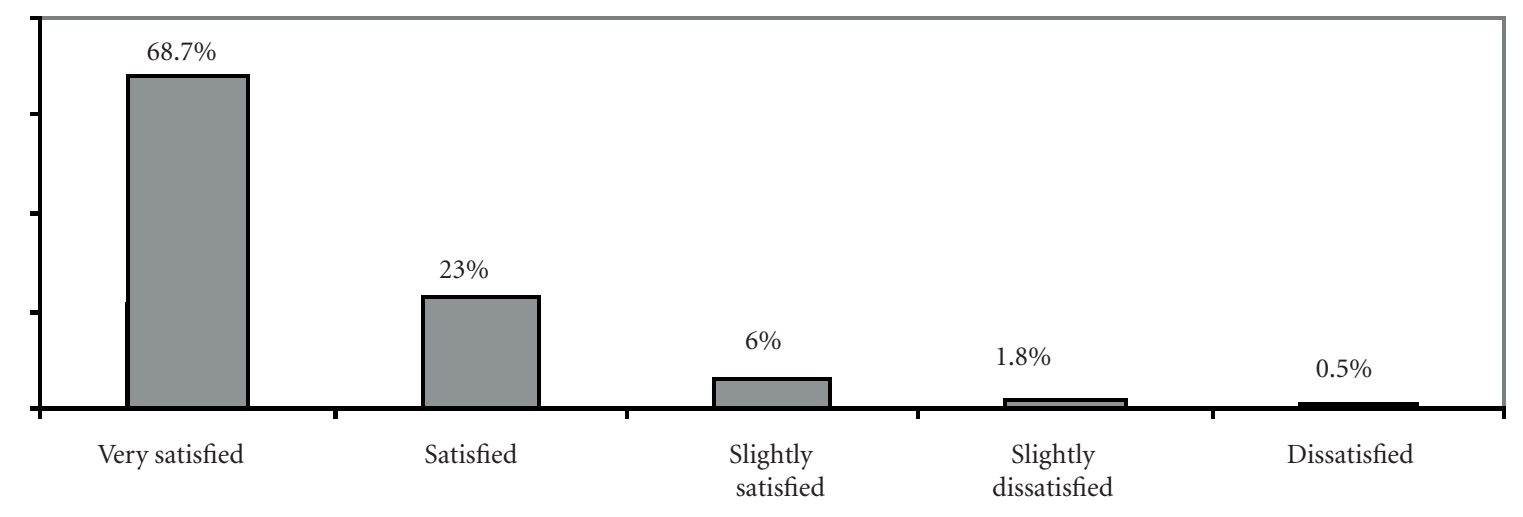

Fig. 4. Patient satisfaction with the immediate outcome of interventional pain management procedure $(s)$

for solid food and two hours for clear liquid, except for trigger point injections. None of the patients vomited or aspirated during interventional pain management procedures.

\section{Patient Satisfaction}

Four hundred forty-two patients answered the question regarding satisfaction. Four hundred six (91.8\%) were satisfied or very satisfied with the results of interventional pain management procedures. Three hundred four $(68.7 \%)$ patients were very satisfied; $102(23 \%)$ satisfied; 28 (6\%) slightly satisfied. Eight (1.8\%) patients indicated they were unsatisfied with the result of interventional pain management procedures (Fig. 4). There is a significant correlation between decrease of pain score and patient satisfaction $\left(\mathrm{R}^{2}=0.05644, \mathrm{P}<0.0001\right)$. Patients with a higher level of pain relief tended to be more satisfied with the results of their treatment.

\section{Discussion}

Results of the current study indicate that quality assurance techniques can be applied to interventional pain management procedures. A high quality interventional program with high rates of pain relief, low rates of complications and high patient satisfaction can be achieved through efforts to monitor, analyze and adjust the process of care.

Traditionally, studies on efficacy of chronic pain management have focused on long-term effects (16). How- ever, the immediate effect of treatment is also critical for success in the management of chronic pain. Local anesthetics block the sodium channel and inhibit the conduction of pain information into the CNS. Most fluoroscopy-guided interventional pain management procedures deliver local anesthetics, frequently with corticosteroid, to an assumed pain source, such as a nerve root. Immediate pain relief could be expected if both clinical diagnosis and needle placement are accurate. Thus, the rate of immediate pain relief should be an indication of the accuracy of the clinical diagnosis as well as the correctness of needle placement. Furthermore, it could also reflect the appropriateness of patient selection. We believe that immediate pain re- 
lief should be the first indicator for the quality of interventional pain management procedures.

Our data showed that $92 \%$ of patients had various degrees of pain relief immediately after procedures with a mean numeric pain score reduction of 4.7. Even though placebo effect plays a role in the results of treatment, the high rate of immediate pain relief in this study reflected our efforts to obtain an accurate diagnosis prior to interventional pain management procedures, strict control of procedure indication, as well as emphasis on the accuracy of needle placement. In interventional pain management procedures, immediate pain relief is usually the result of local anesthetic use, while longterm pain reduction appears to be the result of other components of the treatment, such as the anti-inflammatory effect of steroids for lumbar radicular pain $(17,18)$. A high rate of immediate pain relief could potentially forecast better long-term pain reduction. However, more studies are needed to confirm this hypothesis.

Patient safety has drawn major attention over recent years $(19,20)$. In interventional pain management procedures, many factors can affect the incidence of complications and patient safety. These factors include underlying medical conditions, medications, type of interventional pain management procedures, and the particular drugs injected, physician skill level, and patient preparedness, among others.

Various adverse reactions have been reported in interventional pain management procedures. For epidural steroid injection, these include spinal headache, abscess formation (21), epidural hematoma (22), acute retinal necrosis (23), meningitis, arachnoiditis and inadvertent subdural or subarachnoid injection, temporary quadriplegia (24), spinal cord infarction (25) and cerebellar infarction (26). Serious life threatening side effects can occur for many other interventional pain management procedures.

With the help of strict pre-procedure preparation and continuous monitoring of patients, as described in the methodology section, no major com- plications were noted in our patient series. However, cardiovascular fluctuation was common in our study as has been reported by other investigators (27). The exact causes of cardiovascular fluctuation in this group of patients are not clear. However, age and history of hypertension may play a role. The four patients who developed critical hypertension in our study had a history of hypertension, and the mean age of these four patients was 54.3 years. The mean age of those who developed vasovagal response including bradycardia and hypotension was 40 years. None of these patients had history of hypertension. Because of the small sample (four patients in each group), age difference was not statistically significant. However, it is worthy of future research whether those with hypertension history and old age are more likely to develop critical hypertension and those with no history of hypertension, and young age are more likely to develop vasovagal response.

Ahmed et al (27) performed a survey among 105 pain practices in the US. In the 12 months prior to survey completion, $72 \%$ of responding pain physicians treated patients with vasovagal responses with a mean of 7.3 reactions occurring per practice (median 5, range 1-50). Six percent of respondents have dealt with cardiopulmonary arrest in the previous year, with a mean of 4.3 affected patients per practice. In the current study, four patients developed serious hypertension over 200/100 mm Hg. Two patients had bradycardia and hypotension. Two patients developed hypotension without bradycardia. Due to continuous monitoring, we were able to abort the pain procedure and start treating bradycardia and hypotension immediately. None of these patients developed a life threatening event.

To help avoid major complications, the authors have established the following rules: 1) avoid interventional pain procedures for those patients with serious cardiovascular risks, such as recent chest pain, MI, severe heart failure, respiratory failure, uncontrolled hypertension or hypotension; 2) continuous monitoring of heart rate, EKG and oxygen saturation throughout the proce- dure; 3) continuous verbal communication with patients during the procedure to disclose any symptoms such as dizziness, shortness of breath or severe pain; 3) immediate discontinuation of the procedure if there is any sign of cardiovascular instability, such as uncontrolled hypertension, bradycardia, hypotension, hypoxia and; 4) immediate treatment of any adverse reaction. By continuous monitoring of patients and immediate response, all four cases of vasovagal responses were stabilized within a few seconds to minutes. No one developed cardiovascular arrest.

Ahmed et al (27) reported various rates of cardiovascular monitoring during interventional pain management procedures across the nation. According to Ahmed et al (27), $89 \%$ to $92 \%$ of patients had blood pressure monitoring and $79 \%$ to $87 \%$ patients had pulse oximetry monitoring during cervical epidural steroid injections and facet joint blocks. Due to the potential seriousness of vasovagal reaction and cardiopulmonary arrest induced by interventional pain management procedures, it is suggested that all the patients undergoing spine procedures have continuous blood pressure, heart rate and pulse oximetry monitoring. An IV line should be inserted for high risk patients or procedures more likely to develop cardiovascular instability, such as cervical or lumbar sympathetic blocks.

Guidelines for interventional pain management procedures are needed to improve patient safety, decrease adverse reactions, and to increase the quality of care for patients undergoing interventional pain management procedures. Guidelines for peri-procedural protocols including NPO status, peri-operative monitoring and post-procedural care for all the interventional pain management procedures are essential.

Patient satisfaction is another critical indicator of the quality of health care. A positive relationship between the degree of patient satisfaction and the overall evaluation of the quality of health care has been reported (28). In our study, we utilized patient satisfaction as an outcome measure of quality. It was found that patient satisfaction is correlated with the overall pain decrease 
after interventional pain management procedures; patients with greater pain relief after a procedure tend to be more satisfied with their treatments. There is no correlation between pain/discomfort during the procedures and patient satisfaction. The result suggests that patients are likely to forgive the discomfort or pain during the procedure as long as the treatment can provide adequate pain relief when it is finished. However, there is a negative relationship between pain during the procedure and pain relief after the procedures. Patients who experience more pain during the procedure are more likely to have less pain relief after the treatment. These results require practitioners to enhance their skills and reduce the pain during interventional pain management procedures in order to achieve a better treatment outcome.

The current study used pain relief, adverse reaction/patient safety and patient satisfaction as the main indicators for quality assurance for interventional pain management procedures. The study found that quality of interventional pain management procedures can be measured through systematic monitoring. A high quality interventional pain management procedures program, with a high rate of pain relief, a low rate of complications, and high patient satisfaction can be achieved through appropriate selection of patients, pre-procedural preparation, skillful execution of interventional pain management procedures, continuous monitoring of patients and post procedural care. However, results of the current study only reflect the quality of the interventional pain management program in one university teaching hospital pain center. Major differences most likely exist among various practices in the quality of patient care for interventional pain management procedures across the nation (27). Currently, no universal guidelines are available for the measurement of quality of interventional pain management procedures. We believe such guidelines are needed. The quality assurance methods described here may be used to monitor long-term efficacy and satisfaction of interventional pain management procedures. Ultimately, this approach may improve long-term efficacy, reduce complications, and enhance patient satisfaction.

\section{Conclusion}

Quality of interventional pain management procedures can be monitored. Because no other quality assurance study on interventional pain management procedures were found through the literature search, data comparison is not possible until more data is published. However, the results of the current study indicate that quality assurance of interventional pain management procedures in terms of immediate pain relief following the procedure, low complication rate, and high patient satisfaction can be achieved through application of a quality assurance program.

\section{Author Affiliation:}

\section{YiLi Zhou, MD, PhD}

Courtesy Clinical Assistant Professor

Department of Anesthesiology

University of Florida

Comprehensive Pain Management of

North Florida

10303 SW $48^{\text {th }}$ Place

Gainesville, FL 32608

Email : Yilizhoumed@yahoo.com

\section{Fred A. Furgang, MD}

Acting Director

University of Miami Pain Center

Department of Anesthesiology

Perioperative Medicine and Pain

Management

University of Miami School of

Medicine

1611 NW $12^{\text {th }}$ Av

Miami, FL 33136

Email: FFurgang@med.miami.edu

YanPing Zhang, PhD

Department of Anesthesiology

Perioperative Medicine and Pain

Management

University of Miami School of

Medicine

1011 NW, 15th ST

Gautier Building, \#419

Miami, FL 33136

Email: yzhang@med.miami.edu

\section{REFERENCES}

1. Roe MT. Success stories: how hospitals are improving care. Am Heart J 2004; 148:S52-S55.

2. Baier RR, Gifford DR, Patry G, Banks SM, Rochon T, DeSilva D, Teno JM. Ameliorating pain in nursing homes: A collaborative quality-improvement project. I Am Geriatr Soc 2004; 52:1988-1995.

3. Winn PA, Dentino AN. Effective pain management in the long-term care setting. J Am Med Dir Assoc 2004; 5:342352.

4. Davis MP, Walsh D. Cancer pain: how to measure the fifth vital sign. Cleve Clin J Med 2004; 71:625-632.

5. Rosenquist RW, Rosenberg J. Postoperative pain guidelines. Reg Anesth Pain Med 2003; 28:279-288.

6. Urion L, Bayoumeu F, Jandard C, Fontaine B, Bouaziz H. Quality assurance program for pain management after obstetrical perineal injury. Ann Fr Anesth Reanim 2004; 23:1050-1056.

7. Anonymous Clinical practice guidelines for quality palliative care. Kans Nurse 2004; 79:16-20.

8. Bales DM. The Kansas living initiatives for end-of-life care. J Pain Palliat Care Pharmacother 2003; 17:71-82.

9. Mularski RA. Pain management in the intensive care unit. Crit Care Clin 2004; 20:381-401.

10. Langer HE, Ehlebracht-Konig I, Mattussek S. Quality assurance of rheumatologic patient education. Z. Arztl Fortbild Qualitatssic 2003; 97:357-363.

11. MacLean CH, Saag KG, Solomon DH, Morton SC, Sampsel S, Klippel JH. Measuring quality in arthritis care: methods for developing the Arthritis Foundation's quality indicator set. Arthritis Rheu 2004; 51:193-202.

12. Emons MF. Applying the PAIN indicators in a managed care setting to improve pain management. Manag Care 2003; 12:18-21.

13. Thomas SH, Andruszkiewicz LM. Ongoing visual analog score display improves Emergency Department pain care. J Emerg Med 2004; 26:389-394.

14. Hulsemann JL, Mattussek S, Hennig H, Stucki G. Quality management of treatment of rheumatoid arthritis in a rheumatological setting. Z. Arztl Fortbild Qualitatssic 2003;97:383-390.

15. Manchikanti L. The growth of interventional pain management in the new millennium: A critical analysis of utilization in the medicare population. Pain Physician 2004; 7:465-482.

16. Boswell MV, Shah RV, Everett CR, Sehgal N, Mckenzie-Brown AM, Abdi S, Bowman RC, Deer TR, Datta S, Colson 
JD, Spillane WF, Smith HS, Lucas-Levin LF, Burton AW, Chopra P, Staats PS, Wasserman RA, Manchikanti L. Interventional techniques in the management of chronic spinal pain: Evidencebased practice guidelines. Pain Physician 2005; 8:1-47.

17. Vad VB, Bhat AL, Lutz GE, Cammisa F. Transforaminal epidural steroid injections in lumbosacral radiculopathy: a prospective randomized study. Spine 2002; 27:11-16.

18. Watts RW, Silagy CA. A meta-analysis on the efficacy of epidural corticosteroids in the treatment of sciatica. Anaesth Intensive Care 1995; 23:564-569.

19. Adamski P. Revealed: 2006 national patient safety goals. Nurs Manag 2005; 36:10.

20. Vila H, Jr., Smith RA, Augustyniak MJ, Nagi PA, Soto RG, Ross TW, Cantor AB, Strickland JM, Miguel RV. The efficacy and safety of pain management before and after implementation of hospital- wide pain management standards: is patient safety compromised by treatment based solely on numerical pain ratings? Anesth Analg 2005; 101:47480.

21. Puehler W, Brack A, Kopf A. Extensive abscess formation after repeated paravertebral injections for the treatment of chronic back pain. Pain 2005; 113:427429.

22. Ain RJ, Vance MB. Epidural hematoma after epidural steroid injection in a patient withholding enoxaparin per guidelines. Anesthesiology 2005; 102:701703.

23. Browning DJ. Acute retinal necrosis following epidural steroid injections. Am J Ophthalmol. 2003; 136:192-194.

24. Karasek M, Bogduk N. Temporary neurologic deficit after cervical transforaminal injection of local anesthetic. Pain Med 2004; 5:202-205.

25. Ludwig MA, Burns SP. Spinal cord in- farction following cervical transforaminal epidural injection: a case report. Spine 2005; 30:E266-268.

26. Tiso RL, Cutler T, Catania JA, Whalen K. Adverse central nervous system sequelae after selective transforaminal block: the role of corticosteroids. Spine J 2004; 4:468-474.

27. Ahmed SU, Tonidandel W, Trella J, Martin NM, Chang Y. Peri-procedural protocols for interventional pain management techniques: A survey of U.S. pain centers. Pain Physician 2005; 8:181186.

28. Gonzalez N, Quintana JM, Bilbao A, Escobar A, Aizpuru F, Thompson A, Esteban C, San Sebastian JA, La Sierra ED. Development and validation of an inpatient satisfaction questionnaire. Int J Qua Health Care 2005; 17:465-72. 